\title{
Imaging of Acute Respiratory Distress Syndrome
}

\author{
Sarah Sheard MBBS, Praveen Rao MRCP FRCR, and Anand Devaraj MD MRCP FRCR
}

\author{
Introduction \\ Plain Chest Radiography \\ Exudative or Acute Phase (1-7 Days) \\ Proliferative or Intermediate Phase (8-14 Days) \\ Fibrotic or Late Phase ( $>15$ Days) \\ Computed Tomography in ARDS \\ The Early Phase \\ The Late Phase and Appearances in Long-Term Survivors of ARDS \\ CT in ARDS: Can It Identify Etiology? \\ CT in ARDS: A Tool to Identify Complications \\ CT in ARDS: Can It Be Used to Direct Ventilation? \\ CT as a Means of Prognosticating in ARDS \\ Summary
}

\begin{abstract}
Chest radiography and computed tomography (CT) have a crucial role to play in the diagnosis and management of acute respiratory distress syndrome (ARDS). The identification of pulmonary opacification is a requirement for the definition of ARDS on the chest radiograph, while CT has a role to play, not only in the diagnosis of ARDS, but also in the identification of complications. This paper reviews the radiological appearances of ARDS that have been documented for some time, and also more recent research that has identified a role for $\mathrm{CT}$ in directing ventilation and in prognostication. Key words: acute respiratory distress syndrome; ARDS; computed tomography; CT; imaging; intensive care; prognosis; etiology. [Respir Care 2012;57(4):607-612. (C) 2012 Daedalus Enterprises]
\end{abstract}

\section{Introduction}

Acute respiratory distress syndrome (ARDS) is a severe form of acute lung injury (ALI), which has up to a $50 \%$ risk of mortality. ${ }^{1}$ Pathologically, ARDS is characterized by diffuse alveolar damage, which is created by abnormal

\footnotetext{
The authors are affiliated with the Department of Radiology, St George's Hospital, London, United Kingdom.

The authors have disclosed no conflicts of interest.

Correspondence: Anand Devaraj MD MRCP FRCR, Department of Radiology, St George's Hospital, Blackshaw Road, London SW17 0QT, United Kingdom.
}

DOI: $10.4187 /$ respcare.01731 alveolar-capillary permeability, resulting in an influx of protein and fluid into the alveoli and interstitial spaces. This process is caused by alveolar injury secondary to one (or more) of many precipitating insults, including sepsis and toxic inhalational injuries. Ensuing fibrin deposition and collagen formation can lead to pulmonary fibrosis.

ARDS and ALI exist on the same spectrum of clinical features, pathophysiology, and radiographic appearances. The term ARDS is reserved for the most severe end of this spectrum, when the $\mathrm{P}_{\mathrm{aO}} / \mathrm{F}_{\mathrm{IO}_{2}}$ is $<200 \mathrm{~mm} \mathrm{Hg}$, as opposed to $<300 \mathrm{~mm} \mathrm{Hg}$ in ALI. In this sense, all ARDS patients have ALI, but all ALI patients do not have ARDS. ${ }^{2}$ The definition of both ARDS and ALI, however, requires the presence of bilateral infiltrates on the chest radiograph. ${ }^{2}$

Imaging thus plays an important role in the diagnosis of ARDS and may also provide clues as to the etiology and 
chronicity of the condition. At the outset of this paper, it should be stressed that radiological features by themselves are nonspecific, and correlation with clinical findings is mandatory. For example, the etiology of ARDS may be classified as direct (pulmonary ARDS) or indirect (nonpulmonary ARDS), and these categories in turn may have distinct radiological appearances. However, in clinical practice complex patients with ARDS frequently have a combination of pulmonary and nonpulmonary pathologies, and the exact cause can be difficult to pinpoint by radiological means alone. ${ }^{3}$ As well as establishing the diagnosis, imaging has a role to play in the monitoring of ARDS and in identifying clinical complications. It is also increasingly being shown to provide prognostic information. This paper will review the role of imaging (principally computed tomography $[\mathrm{CT}]$ ) in diagnosing and managing patients with ARDS.

\section{Plain Chest Radiography}

The plain chest radiograph is a valuable test that can support the diagnosis, confirm the position of tubes and lines, monitor progression of pulmonary disease, and detect complications in ARDS. The plain radiographic appearances in ARDS vary depending on the stage of the disease, but these features are usually stereotypical irrespective of the cause. Although there is considerable overlap, the radiographic stages of the disease broadly correlate with the histopathological stages outlined below.

\section{Exudative or Acute Phase (1-7 Days)}

There is usually a radiographic latent period in the first 24 hours following the initial insult that causes ARDS, and the chest radiograph is frequently normal. ${ }^{4}$ The exception is if ARDS is triggered by a direct lung injury (for example pneumonia), in which case consolidation may be observed. There is rapid deterioration in the subsequent $24-$ 72 hours. Injury to the endothelium results in increased capillary permeability and the influx of protein-rich fluid into the alveolar space and interstitium, which promotes pulmonary edema. Air space and interstitial opacities on the chest radiograph in ARDS are predominantly bilateral and symmetrical. ${ }^{5}$ Cardiogenic pulmonary edema is the main radiographic differential diagnosis, but the lack of temporal change; absence of cardiomegaly, septal lines, or pleural fluid; and the presence of peripheral alveolar opacities all favor ARDS. In practice, ARDS and cardiogenic pulmonary edema can coexist, and attempting to discriminate between the two is often difficult using chest radiography alone.
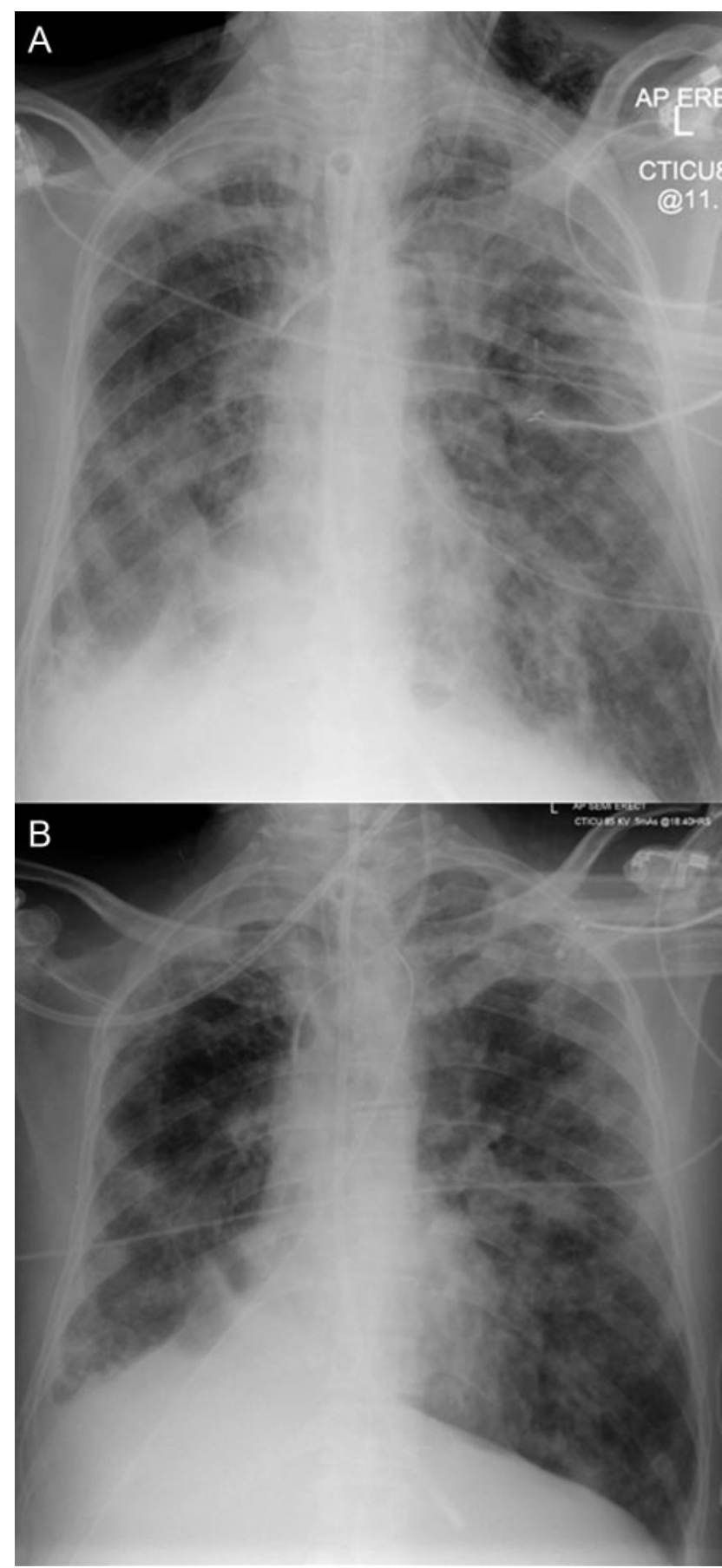

Fig. 1. A: Chest radiograph of patient with ARDS shows bilateral infiltrates. There is bilateral consolidation and a right pleural effusion. B: Chest radiograph of the same patient shows persistent bilateral infiltrates after 7 days.

\section{Proliferative or Intermediate Phase (8-14 Days)}

After the rapid development of radiographic changes in the acute phase of ARDS, the appearances usually stabilize and remain static for a variable length of time (Fig. 1). 
During the intermediate phase, diffuse coarse reticular opacities may develop on the chest radiograph, although this does not imply irreversible fibrosis, as the opacities may resolve. It is important to be aware of this stable period in the radiographic appearances of the disease, as new air space opacities are likely to represent superadded infection or other complications outside the natural history of ARDS.

\section{Fibrotic or Late Phase ( $>15$ Days)}

If the patient survives the acute phase of ARDS, most radiographic abnormalities begin to resolve in the late phase. The speed and duration of recovery are variable and depend on various factors, including comorbidities. Indeed, the chest radiograph in the final phase may range from completely normal to widespread coarse reticular opacities. ${ }^{4}$

\section{Computed Tomography in ARDS}

Since the CT features of ARDS were first described, ${ }^{6}$ the use of CT in ARDS has become widespread both for clinical and research purposes. CT has been shown to be helpful, not only as a confirmatory and problem-solving tool, but emerging studies have shown the potential for classifying and prognosticating ARDS. Of course, the benefits of CT imaging in the clinical setting have to be weighed against the practicalities and risks associated with transporting a patient from the intensive care unit to the radiology department.

\section{The Early Phase}

The classical CT appearance of acute phase ARDS is that of opacification that demonstrates an anterio-posterior density gradient within the lung, with dense consolidation in the most dependent regions, merging into a background of widespread ground-glass attenuation and then normal or hyperexpanded lung in the non-dependent regions (Fig. 2). Ground-glass opacification on CT is a non-specific sign that reflects an overall reduction in the air content of the affected lung. In the case of acute ARDS, this is likely to represent edema and protein within the interstitium and alveoli. Another important observed feature in acute ARDS is bronchial dilatation within areas of ground-glass opacification (Fig. 3). While this may be an indicator of the development of early fibrosis (so-called traction bronchiectasis), the sign cannot be taken as conclusive proof of fibrosis because the reversal of bronchial dilatation in the later stages of ARDS is a well recognized phenomenon. ${ }^{7}$

The theory behind the inhomogeneity of appearances in ARDS is that the increased weight of overlying lung causes compressive atelectasis posteriorly, which produces dense opacification. The theory is supported by the fact that when the position of a patient with ARDS is changed from supine to prone, the density gradient quickly redistributes accordingly. ${ }^{8}$

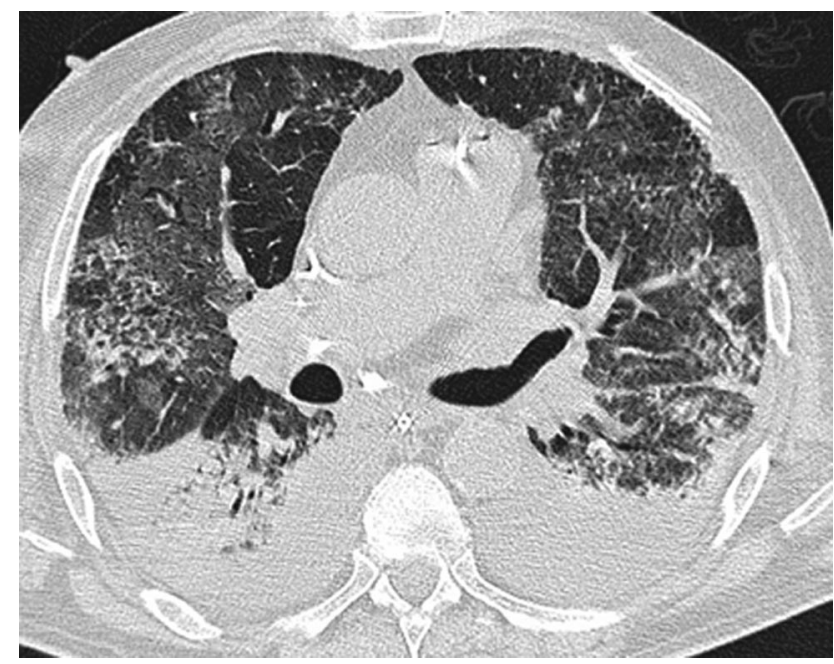

Fig. 2. Computed tomogram of a patient with ARDS shows bilateral dense dependent consolidation, with areas of ground-glass opacification and normal lung in the non-dependent lung.

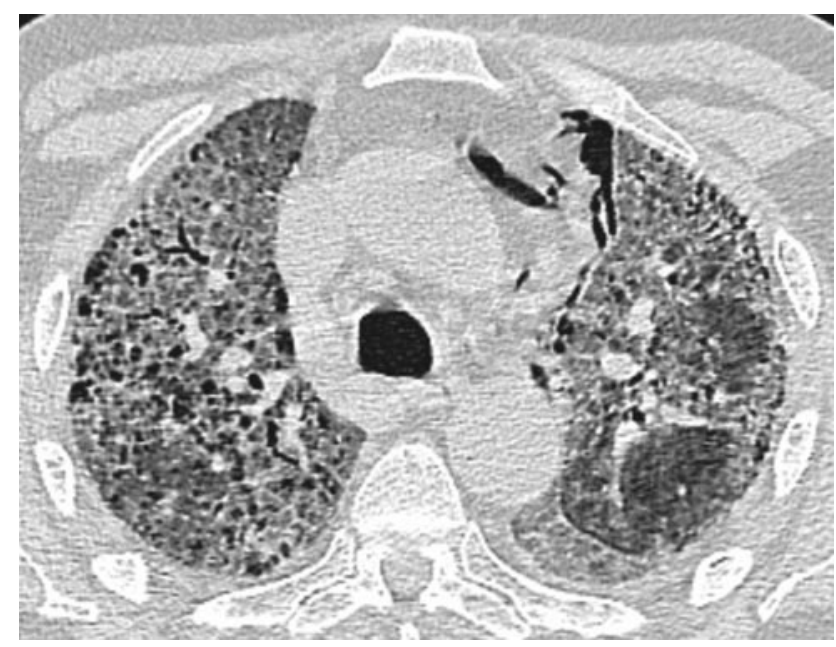

Fig. 3. Computed tomogram in ARDS shows bilateral reticulation and ground-glass opacification, containing areas of bronchial dilatation in the upper lobes. In the acute phase of ARDS, bronchial dilatation may indicate fibrosis or may be reversible. Note also the pneumomediastinum identified by computed tomogram.

In the nondependent portions, lung may be of normal attenuation, or it may be even lower if the patient is being mechanically ventilated. The identification of dense consolidation in nondependent areas of the lung should always alert the clinician to the possibility of infective consolidation, either from a pre-existing pneumonia (which may have been the precipitating condition in ARDS) or new ventilation-associated pneumonia (Fig. 4).

\section{The Late Phase and Appearances in Long-Term Survivors of ARDS}

After the acute phase, CT appearances are variable. Although complete resolution of abnormalities may occur, 


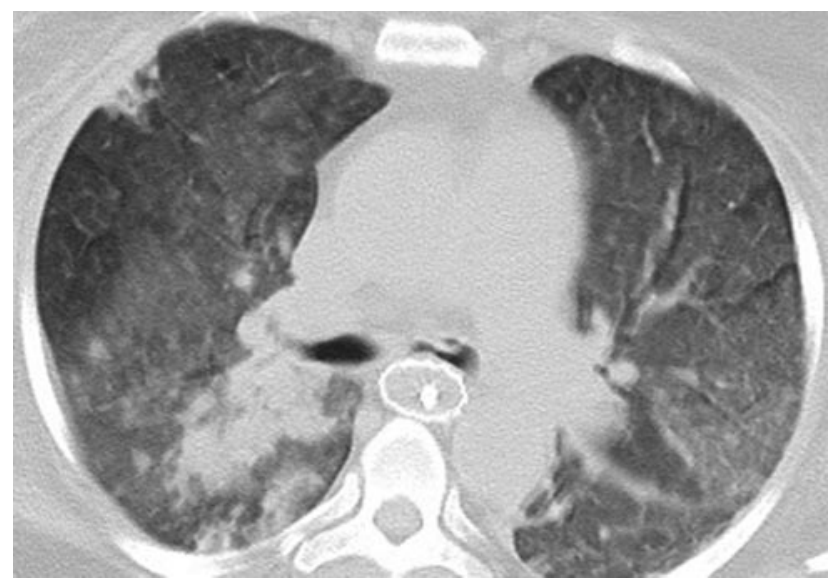

Fig. 4. Computed tomogram of the mid zones of a patient with ARDS shows bilateral ground-glass opacification. Note the presence of non-dependent consolidation in the right lower lobe, which raises the possibility of superadded infection. The esophageal stent is incidental.

the more typical later stage CT appearances are that of a coarse reticular pattern and ground-glass opacification in the anterior (nondependent) part of the lungs..$^{9,10}$ Drawing conclusions about the meaning of appearances in ARDS survivors is always limited by the lack of accompanying histopathology in most patients. However, in this setting, it is likely that the ground-glass opacities represent areas of fine fibrosis, which are too small to be resolved on CT. It can also be speculated that fibrosis develops in these patients as a sequelae of barotrauma secondary to mechanical ventilation in the nondependent lung, with consolidation and atelectasis conferring relative protection on the more dependent regions.

A more recent study has also reported a less classical distribution of disease in ARDS survivors. Masclans et al ${ }^{11}$ found that $76 \%$ of patients had abnormalities on highresolution $\mathrm{CT}$ at 6 months follow-up, which were typically areas of reticular and ground-glass opacification. However, the majority of patients had a diffuse distribution of disease, with only approximately a third having the more classical exclusively anterior changes. A smaller number $(18 \%)$ were also reported to have exclusively posterior abnormalities. The study also reiterated the finding that airways disease (presumed to mean traction bronchiectasis) was common, again implying underlying lung fibrosis in these patients.

Pulmonary cysts of varying sizes and bullae are also features of the later stages of ARDS and probably develop as a result of prolonged ventilation ${ }^{12}$ (Fig. 5). However, pulmonary cysts are also know to arise secondary to pneumonia and do not necessarily have to be associated with an episode of prolonged ventilation. ${ }^{10}$

Small but significant differences have been described at follow-up between different groupings of ARDS patients.

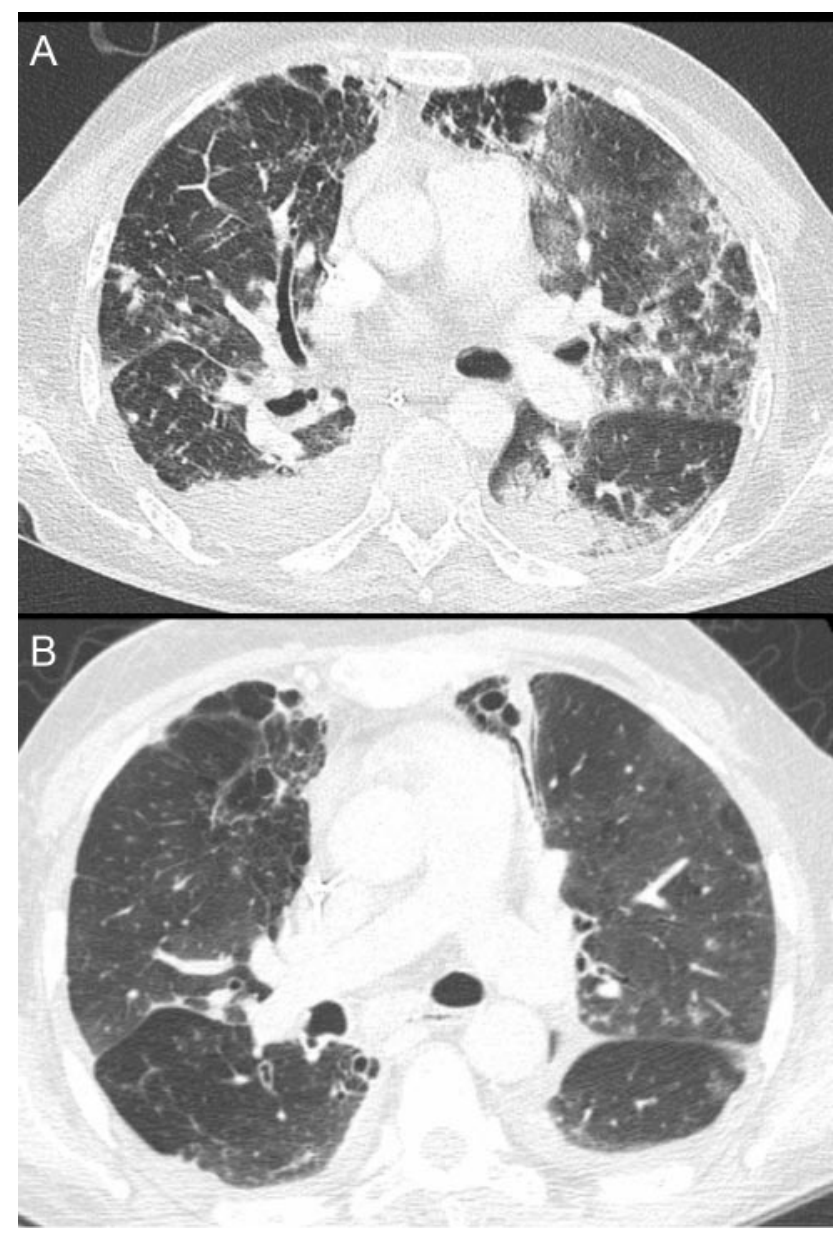

Fig. 5. A: Computed tomogram shows bilateral dependent consolidation in a patient with ARDS, as well as ground-glass opacities in the non-dependent lung. B: Follow-up computed tomogram after 1 year shows resolution of the consolidation and groundglass opacification with cyst formation in the anterior left lung.

Higher levels of lung fibrosis are present on CT in patients who spent the greatest length of time on mechanical ventilation, and who had the highest PEEPs. ${ }^{9}$ Furthermore, by dividing patients strictly into groups of pulmonary and nonpulmonary ARDS it has been shown that pulmonary ARDS survivors are more likely to have pulmonary fibrosis on CT. ${ }^{13}$ It has been speculated that this is because patients with pulmonary ARDS have less lung susceptible to alveolar recruitment and thus are more prone to ventilator-induced lung injury.

\section{CT in ARDS: Can It Identify Etiology?}

The etiology of ARDS can have implications for clinical management because the mechanical ventilation of pulmonary and extra-pulmonary ARDS patients may differ. Lung compliance is lower in patients with pulmonary ARDS, and increasing PEEP tends to reduce lung compli- 
ance further still. Conversely, in extra-pulmonary ARDS, increasing the PEEP can have a desirable effect, as it tends to increase lung compliance. ${ }^{14}$

With this in mind, it could be of clinical use if CT could determine whether the cause of ARDS is intra- or extrapulmonary. Studies have shown that a typical CT pattern (symmetrical ground-glass opacification, with an anteroposterior gradient from dense consolidation to normally aerated lung) is more commonly associated with extrapulmonary ARDS. ${ }^{15,16}$ In pulmonary ARDS the distribution of lung changes are more likely to be asymmetrical, with a mixed picture of ground-glass opacification, nondependent dense consolidation, and parenchymal cysts. ${ }^{15,16}$ Other features, such as pleural effusions and air bronchograms, are said to be equally common to both types of ARDS. ${ }^{16}$

However, whether these associations are strong enough to reliably use CT to determine the cause of ARDS is debatable. In clinical practice it is often difficult to attribute a single cause to ARDS in the first place. In summary, CT cannot be routinely recommended as a method of identifying the cause of ARDS, but rather as a tool to confirm the diagnosis and its complications.

\section{CT in ARDS: A Tool to Identify Complications}

CT imaging is not required to fulfill the diagnostic criteria for ARDS, but given the diffuse opacification that is characteristic of the condition, the chest radiograph can be limited in terms of identifying complications, and this is where cross-sectional imaging with CT can be of assistance.

In an ARDS patient who is deteriorating, CT can detect various ventilation-associated complications and foci of infection that may not be apparent on the antero-posterior, supine, intensive care unit chest radiograph. Sequelae of mechanical ventilation that may be more easily detected on CT include pneumonia, abscesses, pneumothorax, pneumomediastinum, and pulmonary interstitial emphysema. The position of the endotracheal tube can also be established, and lung collapse due to malpositioning can be identified.

\section{CT in ARDS: Can It Be Used to Direct Ventilation?}

The mainstay of management in ARDS is mechanical ventilation. This life-saving therapy is also an invasive one, with potential to cause barotrauma and long-term damage to the lungs. Recruitment is a term that refers to the number of previously collapsed acini that can be inflated for a given pressure. Increasing PEEP recruits more lung at the higher pressure end of the spectrum. However, increased ventilation pressure can cause barotrauma to the lung that would have successfully been ventilated at lower pressures. This means that there is a trade-off between recruiting more acini for gas exchange and causing ventilator-related lung injury. On CT, collapsed lung can be readily identified, and in the case of ARDS this is likely to be dependent lung, collapsed under the increased weight of the overlying lung. Gattinoni et $\mathrm{al}^{17}$ have suggested performing limited CT of the lungs, with just 2 or 3 slices each on inspiration, expiration, and with different PEEPs, as a means of guiding ventilator settings. By acquiring CT images at different ventilator settings, the assessment of the potential for recruitment can be made by identifying the extent of atelectatic lung and its response to ventilation. Additionally, even with these limited sections, CT may provide the ability to distinguish atelectatic lung from areas of consolidation that would not be amenable to recruitment. ${ }^{18}$

\section{CT as a Means of Prognosticating in ARDS}

Limited studies have examined whether CT findings in early ARDS can predict long-term outcome in individuals. Ichikado et al measured the extent of disease on CT studies performed within one week of the onset of ARDS, and found that the presence of fibrosis (indicated by groundglass opacification, traction bronchiectasis, and honeycombing) was a strong independent predictor of mortality. ${ }^{19}$ The presence of traction bronchiectasis was also found to be a predictor of poor outcome in another study, along with disease affecting over $80 \%$ of the lungs, and signs of elevated right heart pressure (pulmonary artery dilatation and an enlarged right ventricle). ${ }^{20}$ This study also suggested that patients who have a pure consolidative pattern of disease in the early stages have a better chance of survival. The implication of this observation is that, because consolidation is not a marker of fibrotic change, there is a more favorable outcome for this group of patients, thus indirectly supporting the finding that early fibrosis in ARDS is associated with a higher risk of death.

\section{Summary}

The role of the chest radiograph and $\mathrm{CT}$ in the diagnosis and management of ARDS is an important one. Information from the plain radiograph not only forms one of the diagnostic criteria, but it can easily provide ongoing information about the clinical state of the ARDS patient. The role of CT has been vital to the increasing understanding of the disease process in ARDS, and it continues to be an enlightening research tool. In the clinical setting, CT is a recognized and effective means of confirming the diagnosis of ARDS, although it is perhaps most readily used for identifying complications. Although less commonly used for other purposes, it also has the potential to determine ventilation strategies and prognosis. 


\section{Imaging of Acute Respiratory Distress Syndrome}

\section{REFERENCES}

1. Brun-Buisson C, Minelli C, Bertolini G, Brazzi L, Pimentel J, Lewandowski $\mathrm{K}$, et al. Epidemiology and outcome of acute lung injury in European intensive care units. Results from the ALIVE study. Intensive Care Med 2004;30(1):51-61.

2. Bernard GR, Artigas A, Brigham KL, Carlet J, Falke K, Hudson L, et al. The American-European Consensus Conference on ARDS. Definitions, mechanisms, relevant outcomes, and clinical trial coordination. Am J Respir Crit Care Med 1994;149(3 Pt 1):818-824.

3. Kobayashi H, Itoh T, Sasaki Y, Konishi J. Diagnostic imaging of idiopathic adult respiratory distress syndrome (ARDS)/diffuse alveolar damage (DAD) histopathological correlation with radiological imaging. Clin Imaging 1996;20(1):1-7.

4. Desai SR. Acute respiratory distress syndrome: imaging of the injured lung. Clin Radiol 2002;57(1):8-17.

5. Aberle DR, Brown K. Radiologic considerations in the adult respiratory distress syndrome. Clin Chest Med 1990;11(4):737-754.

6. Maunder RJ, Shuman WP, McHugh JW, Marglin SI, Butler J. Preservation of normal lung regions in the adult respiratory distress syndrome. Analysis by computed tomography. JAMA 1986;255(18): 2463-2465

7. Howling SJ, Evans TW, Hansell DM. The significance of bronchial dilatation on $\mathrm{CT}$ in patients with adult respiratory distress syndrome. Clin Radiol 1998;53(2):105-109.

8. Gattinoni L, Pelosi P, Vitale G, Pesenti A, D'Andrea L, Mascheroni D. Body position changes redistribute lung computed-tomographic density in patients with acute respiratory failure. Anesthesiology 1991;74(1):15-23.

9. Desai SR, Wells AU, Rubens MB, Evans TW, Hansell DM. Acute respiratory distress syndrome: $\mathrm{CT}$ abnormalities at long-term followup. Radiology 1999;210(1):29-35.

10. Joynt GM, Antonio GE, Lam P, Wong KT, Li T, Gomersall CD, et al. Late-stage adult respiratory distress syndrome caused by severe acute respiratory syndrome: abnormal findings at thin-section CT. Radiology 2004;230(2):339-346.
11. Masclans JR, Roca O, Munoz X, Pallisa E, Torres F, Rello J, et al. Quality of life, pulmonary function, and tomographic scan abnormalities after ARDS. Chest 2011;139(6):1340-1346.

12. Gattinoni L, Caironi P, Pelosi P, Goodman LR. What has computed tomography taught us about the acute respiratory distress syndrome? Am J Respir Crit Care Med 2001;164(9):1701-1711.

13. Kim SJ, Oh BJ, Lee JS, Lim CM, Shim TS, Lee SD, et al. Recovery from lung injury in survivors of acute respiratory distress syndrome: difference between pulmonary and extrapulmonary subtypes. Intensive Care Med 2004;30(10):1960-1963.

14. Gattinoni L, Pelosi P, Suter PM, Pedoto A, Vercesi P, Lissoni A. Acute respiratory distress syndrome caused by pulmonary and extrapulmonary disease. Different syndromes? Am J Respir Crit Care Med 1998;158(1):3-11.

15. Desai SR, Wells AU, Suntharalingam G, Rubens MB, Evans TW, Hansell DM. Acute respiratory distress syndrome caused by pulmonary and extrapulmonary injury: a comparative CT study. Radiology 2001;218(3):689-693.

16. Goodman LR, Fumagalli R, Tagliabue P, Tagliabue M, Ferrario M, Gattinoni L et al. Adult respiratory distress syndrome due to pulmonary and extrapulmonary causes: CT, clinical, and functional correlations. Radiology 1999;213(2):545-552.

17. Gattinoni L, Caironi P, Cressoni M, Chiumello D, Ranieri VM, Quintel M, et al. Lung recruitment in patients with the acute respiratory distress syndrome. N Engl J Med 2006;354(17):1775-1786.

18. Gattinoni L, Chiumello D, Cressoni M, Valenza F. Pulmonary computed tomography and adult respiratory distress syndrome. Swiss Med Wkly 2005;135(11-12):169-174.

19. Ichikado K, Suga M, Muranaka H, Gushima Y, Miyakawa H, Tsubamoto M, et al. Prediction of prognosis for acute respiratory distress syndrome with thin-section CT: validation in 44 cases. Radiology 2006;238(1):321-329.

20. Chung JH, Kradin RL, Greene RE, Shepard JA, Digumarthy SR. CT predictors of mortality in pathology confirmed ARDS. Eur Radiol 2011;21(4):730-737. 